B. Faye ${ }^{1}$

\section{Ratovonanahary ${ }^{2}$ \\ R. Cherrier ${ }^{3 *}$}

\title{
Effet d'un facteur alimentaire sur la pathologie néonatale : Résultats d'une enquête rétrospective sur la distribution de mangrove aux chamelons en République de Djibouti
}

FAYE (B.), RATOVONANAHARY (M.), CHERRIER (R.).Effet d'un facteur alimentaire sur la pathologie néonatale : Résultats d'une enquête rétrospective sur la distribution de mangrove aux chamelons en République de Djibouti. Revue Élev. Méd. vét. Pays trop., 1993, 46 (3) : 471478

Une enquête rétrospective réalisée dans 23 campements de nomades afars de la région d'Obock (Djibouti) a porté sur les modes d'utilisation traditionnelle de la végétation lagunaire (mangrove à palétuviers) et leur relation avec la pathologie observée, notamment chez les chamelons. Quatre types d'exploitation de la mangrove peuvent être décrits : distribution de branches de palétuvier aux chamelons âgés de moins d'un an dans les campements et pâturage des adultes dans la mangrove ; utilisation permanente de la mangrove associée à une complémentation ; exploitation occasionnelle par émondage ; utilisation permanente sans complémentation. Le risque d'observer des troubles sanitaires (pertes d'appétit, boiterie, paralysie, maladies de peau) est 4,28 fois plus élevé en l'absence de complémentation de la ration de base de mangrove.

Mots clés : Dromadaire - Jeune animal - Alimentation - Palétuvier - Fourrage - Pâturage - Complémentation - Enquête pathologique - Maladie de carence - Maladie de la peau - Trouble locomoteur - Paralysie - Djibouti.

\section{INTRODUCTION}

La mangrove à palétuvier, formation végétale arbustive des lagunes côtières de la mer Rouge, est très carencée en azote et en éléments-traces (oligo-éléments) (5). Pourtant, à Djibouti, elle représente un fourrage de survie pour les animaux d'élevage en cas de sécheresse (9) et en particulier pour les dromadaires.

Les mangroves sont essentiellement composées de Ceriops tagal et d'Avicennia marina (palétuvier), mais seul ce dernier est consommé par les dromadaires (1). A l'intérieur des terres, les ressources fourragères sont rares à l'exception des fourrés monospécifiques à Salvadora persica (1).

Une étude en milieu contrôlé a permis de mettre en évidence la nécessité d'une complémentation en concentré pour réduire les troubles pathologiques d'origine nutri-

1. Laboratoire d'Écopathologie, INRA Theix, 63122 Saint Genès Champanelle, France.

2. Département de Recherches zootechniques et vétérinaires, BP 04, Tananarive 101, Madagascar.

3. UCEC, C/O CIRAD-EMVT, 10 rue Pierre-Curie, 94704 MaisonsAlfort Cedex, France.

* Adresse actuelle : CIRAD-SAR, s/c M. Reboul, BP 853, Tananarive, Madagascar.

Reçu le 22.09.1992, accepté le 25.06.1993. tionnelle chez les animaux nourris principalement avec ce fourrage (8).

En complément de cette étude, une enquête épidémiologique à visée informative a été menée auprès de quelques éleveurs qui utilisent la mangrove comme aliment de base pour le dromadaire. Les objectifs de cette étude étaient, d'une part de réaliser une typologie des élevages camelins en fonction des modes d'exploitation de la mangrove et de quelques paramètres sanitaires et zootechniques, d'autre part d'estimer la relation entre la pathologie des jeunes chamelons et les types d'alimentation.

\section{MATÉRIEL ET MÉTHODES}

\section{Type d'enquête et échantillonnage}

II s'agit d'une enquête descriptive de type rétrospectif réalisée auprès de vingt-trois éleveurs répartis dans cinq campements du district d'Obock (carte 1).

Cette région a été choisie en raison de l'importance des mangroves où les camelins sont presque exclusivement les seuls à subsister $(10,14)$. L'exploitation de ces mangroves concerne les campements d'éleveurs situés dans la plaine de Doumera depuis les premiers contreforts des massifs du Nord-Est dans le district d'Obock jusqu'à la frontière éthiopienne, avec une pointe sur Lahassa à l'intérieur des terres (2).

Les sites ont été retenus en fonction de leur accessibilité. Les éleveurs sont sélectionnés de façon aléatoire, soit aux points d'eau, soit directement dans les campements. Ceux qui ne possèdent pas de dromadaires n'ont pas été retenus.

Les objectifs de l'étude sont : caractériser les élevages camelins en fonction du mode d'utilisation de la mangrove ; mettre en évidence une relation éventuelle entre l'utilisation quasi-exclusive de la mangrove et la pathologie observée sur les animaux, plus particulièrement les jeunes, dans la zone géographique concernée. Dans ce but, le questionnaire comprend deux parties composées chacune de onze questions. La première partie décrit le système d'exploitation de la mangrove. La seconde est relative aux performances zootechniques et sanitaires. Les campements retenus sont Gahéré (10 éleveurs), Khor Anghar (5 éleveurs), Lahassa (4 éleveurs), Kibo (2 éleveurs) et Moulhoulé (2 éleveurs). 


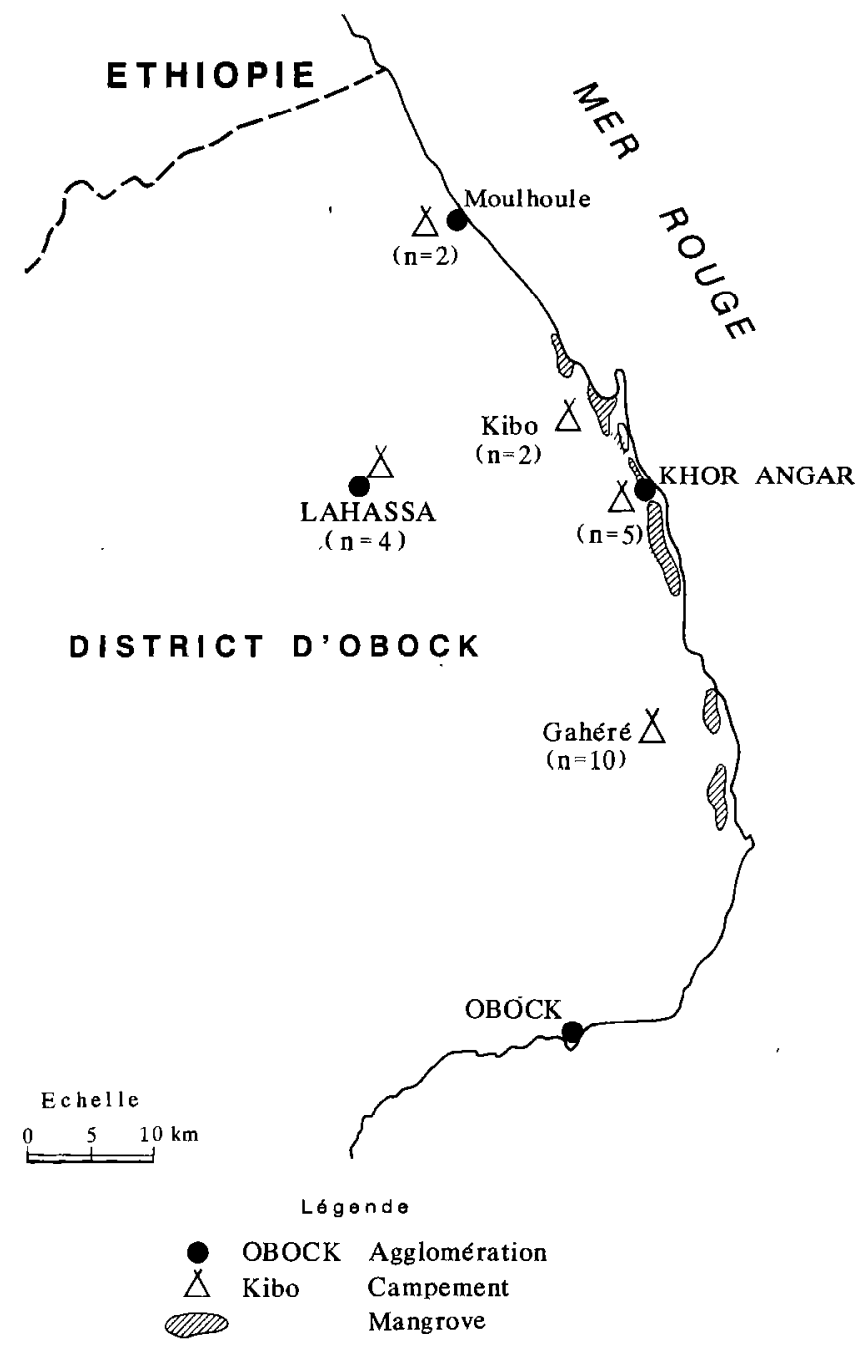

Carte 1: Carte du district d'Obock. Emplacement de la zone d'étude.

\section{Stratégie statistique et traitement informatique}

En épidémiologie, on se situe par définition en milieu non contrôlé. La multiplicité des facteurs en cause implique l'utilisation de méthodes statistiques appropriées. II faut donc insister particulièrement sur la stratégie de mise en œuvre pour étayer les résultats biologiques.

Après un tri des variables (élimination des données redondantes ou trop corrélées et des modalités rares) et la transformation des variables quantitatives en variables qualitatives (classes de quantité), on obtient un tableau homogène comprenant trente-trois variables qualitatives représentant au total 75 modalités (tabl. I et II).

\section{Analyse des données par les méthodes multidimensionnelles (objectif 1 )}

Pour étudier et visualiser les associations des facteurs de risque des pathologies et réaliser une typologie des campements, les analyses multivariées de l'école française des statistiques sont les plus pertinentes (10). Dans cette étude, on a utilisé le logiciel ADDAD (13), bibliothèque de programmes permettant la gestion, l'analyse et l'aide à l'interprétation.

Dans un premier temps, on a constitué un tableau de données comprenant en ligne les vingt-trois campements étudiés et en colonne les trente-trois variables retenues, réparties en deux groupes (tabl. I, II) :

- le groupe 1 qualifie l'utilisation de la mangrove par les éleveurs;

- le groupe 2 qualifie les performances zootechniques et sanitaires des troupeaux .

Ce tableau est transformé en tableau disjonctif complet qui comprend donc au total 75 variables (codées en 0 et 1) réparties en 30 pour le groupe 1 et 45 pour le groupe 2. Ce tableau est ensuite soumis à une analyse factorielle multiple (3) qui permet d'etudier séparement les deux groupes de variables définies plus haut, puis de réaliser une analyse simultanée et pondérée des deux groupes.

La typologie des élevages est réalisée par une méthode de classification automatique (classification ascendante hiérarchique) assortie des aides à l'interprétation (représentation graphique des classes et contribution des variables aux classes). La stratégie statistique est résumée dans la figure 1.

\section{Évaluation du risque sanitaire chez les chamelons (objectif 2)}

D'une manière générale, dans la recherche des facteurs étiologiques d'une maladie dans une population, on compare les différents groupes de sujets ayant une relation, particulière ou non, avec la maladie (malades et sains) et avec le facteur étudié (exposé et non-exposé) (12). L'analyse se rapporte à un tableau de contingence de type 2 par 2 à $n$ strates de facteurs concomitants (tabl. III).

La force de l'association entre la maladie et le facteur d'exposition est mesurée par le risque relatif (RR) qui représente le rapport entre le taux d'incidence de la maladie chez les exposés et le taux d'incidence chez les non-exposés. Les épidémiologistes utilisent également pour exprimer cette relation, l'odd-ratio (OR) qui est le rapport entre le produit du nombre d'animaux malades exposés et du nombre d'animaux non-malades nonexposés sur le produit du nombre d'animaux nonmalades exposés et du nombre d'animaux malades nonexposés (18). 
TABLEAU I Variables qualifiant les modes d'exploitation de la mangrove.

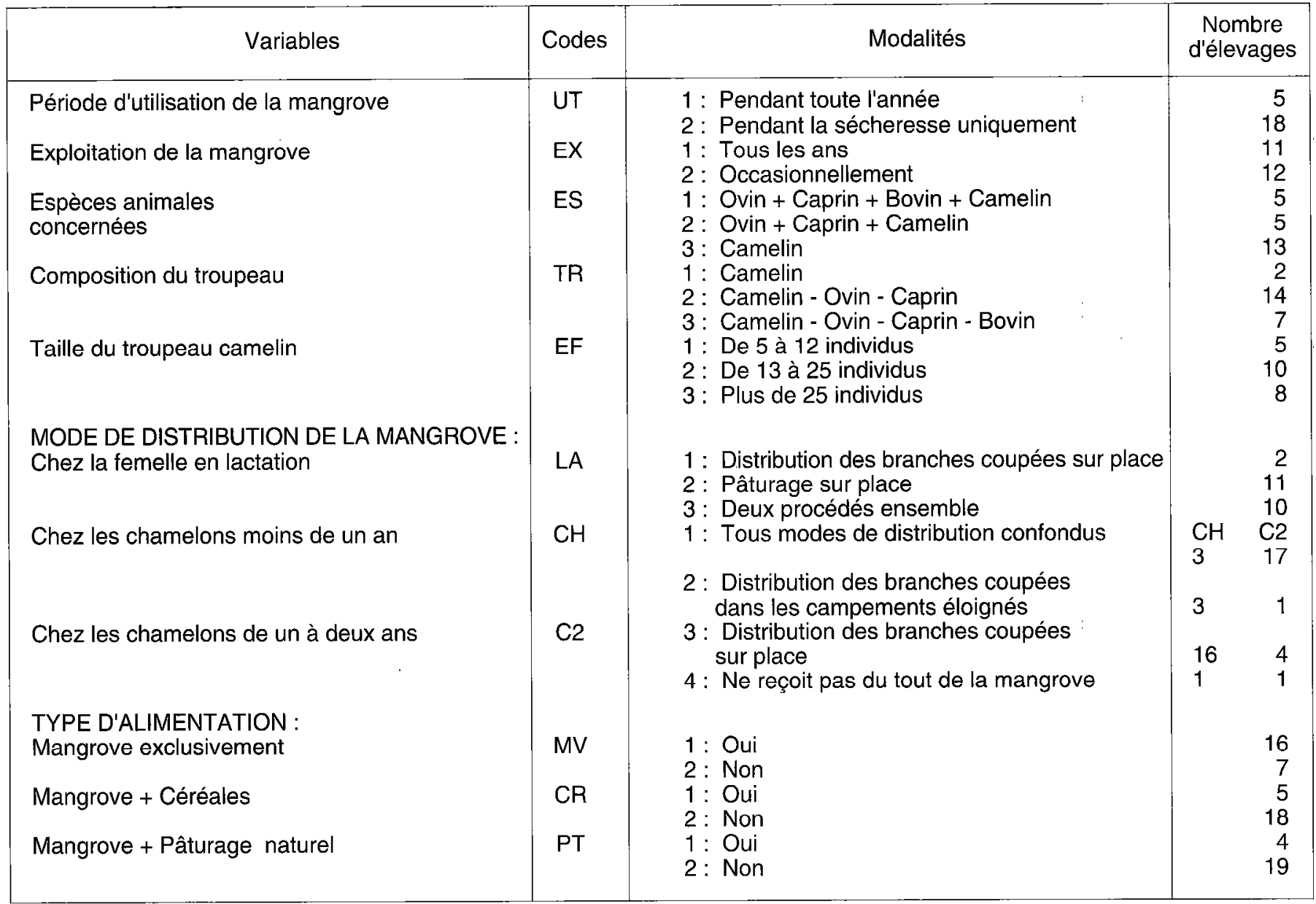

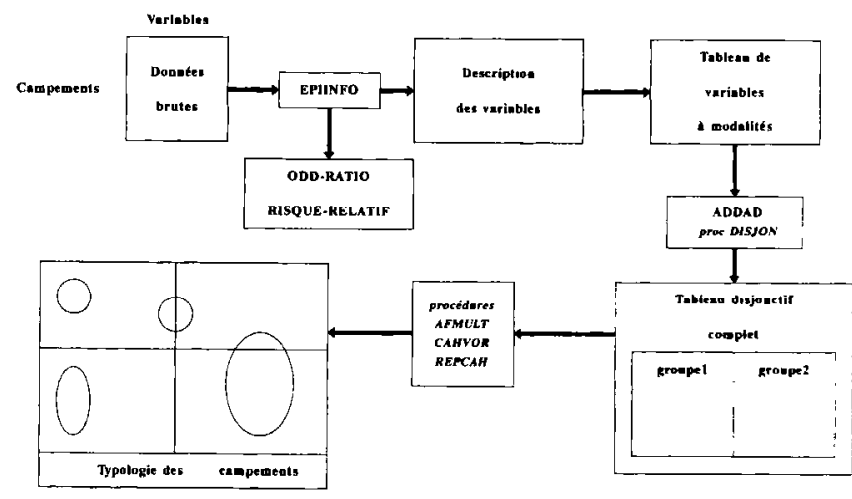

Figure 1 : Schéma de la stratégie d'analyse statistique.

En présence d'un facteur concomitant (dans ce cas, l'âge des chamelons), on a procédé à une analyse ajustée en stratifiant les données selon ce facteur. Dans l'hypothèse de l'uniformité des risques relatifs dans les différentes strates, la valeur de ce risque proposée par MANTELHAENSZEL est égale à :
TABLEAU III Tableau de contingence de type 2 par 2 à $n$ strates de facteurs concomitants.

\begin{tabular}{|c|c|c|c|c|}
\hline \multicolumn{2}{|c|}{} & \multicolumn{3}{|c|}{ Maladie } \\
\cline { 3 - 5 } \multicolumn{2}{|c|}{} & Présence & Absence & Total \\
\hline \multirow{3}{*}{ Exposition } & $\begin{array}{c}\text { Présence } \\
\text { Absence } \\
\text { Total }\end{array}$ & A & B & M1 \\
& N1 & D & M0 \\
& & & No & N \\
\hline
\end{tabular}

\section{$\mathrm{Ai}{ }^{\star} \mathrm{Di} / \mathrm{Ni}$ sur $\mathrm{Ci}{ }^{\star} \mathrm{Bi} / \mathrm{Ni}$}

tel que i prend la valeur de 1 à $n$ strates (17). L'hypothèse d'indépendance entre l'exposition et l'existence de la maladie est vérifiée par le $\chi^{2}$ de MANTEL-HAENSZEL (14).

Pour réaliser ces calculs, on a utilisé le logiciel EPIINFO (Centers for Disease Control, Atlanta, USA, 1988). Dans 
B. Faye M. Ratovonanahary R. Cherrier

TABLEAU II Variables qualifiant les paramètres sanitaires et zootechniques.

\begin{tabular}{|c|c|c|c|}
\hline Variables & Codes & Modalités & $\begin{array}{c}\text { Nombre } \\
\text { d'élevages }\end{array}$ \\
\hline $\begin{array}{l}\text { Mortalité des chamelons } \\
\text { depuis une année }\end{array}$ & MT & $\begin{array}{l}\text { 1: Oui } \\
2: \text { Non }\end{array}$ & $\begin{array}{r}9 \\
14\end{array}$ \\
\hline $\begin{array}{l}\text { PÉRIODES DE MORTALITÉ : } \\
\text { Avant un mois } \\
\text { De un mois à un an } \\
\text { De un à deux ans }\end{array}$ & $\begin{array}{l}\text { PM } \\
\text { PA } \\
\text { PD }\end{array}$ & $\begin{array}{l}1: \text { Oui } \\
2: \text { Non } \\
1: \text { Oui } \\
2: \text { Non } \\
1: \text { Oui }\end{array}$ & $\begin{array}{r}3 \\
20 \\
2 \\
21 \\
16\end{array}$ \\
\hline $\begin{array}{l}\text { CAUSES DE LA MORTALITÉ } \\
\text { DES CHAMELONS : } \\
\text { Sous-alimentation } \\
\text { Ectoparasites } \\
\text { Piqûre des scorpions } \\
\text { Gale } \\
\text { Aphte }\end{array}$ & $\begin{array}{l}\text { SA } \\
\text { EP } \\
\text { SC } \\
\text { GL } \\
\text { AF }\end{array}$ & $\begin{array}{l}1: \text { Oui } \\
2: \text { Non } \\
1: \text { Oui } \\
2: \text { Non } \\
1: \text { Oui } \\
2: \text { Non } \\
1: \text { Oui } \\
2: \text { Non } \\
1: \text { Oui } \\
2: \text { Non }\end{array}$ & $\begin{array}{r}4 \\
19 \\
20 \\
3 \\
12 \\
11 \\
3 \\
20 \\
6 \\
17\end{array}$ \\
\hline $\begin{array}{l}\text { PATHOLOGIE OBSERVÉE } \\
\text { CHEZ LES CHAMELONS : } \\
\text { Paralysie } \\
\text { Maladies de peau } \\
\text { Boiterie } \\
\text { Manque d'appétit }\end{array}$ & $\begin{array}{l}\mathrm{PL} \\
\mathrm{PE} \\
\mathrm{BT} \\
\mathrm{AP}\end{array}$ & $\begin{array}{l}\text { 1: Oui } \\
2: \text { Non } \\
1: \text { Oui } \\
2: \text { Non } \\
1: \text { Oui } \\
2: \text { Non } \\
1: \text { Oui } \\
2: \text { Non }\end{array}$ & $\begin{array}{r}3 \\
20 \\
8 \\
15 \\
7 \\
16 \\
5 \\
18\end{array}$ \\
\hline $\begin{array}{l}\text { CAUSES DE MORTALITÉ } \\
\text { DES ADULTES : } \\
\text { Sous-alimentation } \\
\text { Ectoparasites } \\
\text { Piqûres des scorpions } \\
\text { Aphte }\end{array}$ & $\begin{array}{l}\text { AS } \\
\text { EC } \\
\text { SP } \\
\mathrm{AH}\end{array}$ & $\begin{array}{l}1: \text { Oui } \\
2: \text { Non } \\
1: \text { Oui } \\
2: \text { Non } \\
1: \text { Oui } \\
2: \text { Non } \\
1: \text { Oui } \\
2: \text { Non }\end{array}$ & $\begin{array}{r}12 \\
11 \\
11 \\
12 \\
5 \\
18 \\
4 \\
19\end{array}$ \\
\hline $\begin{array}{l}\text { PARAMÉTRES ZOOTECHNIQUES : } \\
\text { Age de la femelle à la mise } \\
\text { à la reproduction } \\
\text { Nombre de naissances dans } \\
\text { le troupeau dans l'année } \\
\text { Durée de la lactation } \\
\text { Intervalle entre mise-bas } \\
\text { Age de la réforme }\end{array}$ & $\begin{array}{l}\text { SI } \\
\text { NE } \\
\text { LM } \\
\text { IM } \\
\text { RF }\end{array}$ & $\begin{array}{l}1: \text { moins de } 3 \text { ans } \\
2: \geqslant \text { à } 3 \text { ans } \\
1: 2 \text { à } 8 \text { chamelons } \\
2: 9 \text { à } 20 \text { chamelons } \\
3: 21 \text { à } 40 \text { chamelons } \\
1: \text { moins de } 180 \text { jours } \\
2: \text { plus de } 180 \text { jours } \\
1: \leq \text { à } 18 \text { mois } \\
2:>\text { à } 18 \text { mois } \\
1: \text { entre } 14 \text { et } 20 \text { ans } \\
2: \text { plus de } 20 \text { ans. }\end{array}$ & $\begin{array}{r}4 \\
19 \\
11 \\
9 \\
3 \\
5 \\
18 \\
16 \\
7 \\
16 \\
7\end{array}$ \\
\hline
\end{tabular}


le cas présent, il a fallu définir, pour constituer ce tableau de contingence, l'événement "malade" et le "facteur d'exposition".

Sachant que l'existence d'une maladie dans un troupeau n'en exclut pas une autre, on définit l'événement "malade" par la présence d'au moins une des quatres maladies recensées au cours de l'enquête chez les jeunes chamelons élevés dans la région d'étude. On détermine cet événement par une variable synthétique appelée "malade" qui est égale à la somme de la présence des quatre maladies :

$$
\text { malade }=[(\mathrm{PE}=1)+(\mathrm{BT}=1)+(\mathrm{PL}=1)+(\mathrm{AP}=1)]
$$

tel que : $\mathrm{PE}=$ maladie de peau, $\mathrm{BT}=$ boiterie, $\mathrm{PL}=$ paralysie, $\mathrm{AP}=$ manque d'appétit.

L'état carentiel des dromadaires de la région d'Obock est lié autant à la composition minérale des fourrages disponibles (mangrove) qu'au déficit protéique de la ration (8). On peut alors admettre que l'utilisation exclusive de la mangrove, quel que soit le mode de distribution, constitue un facteur d'exposition des chamelons au risque de tomber "malade".

L'apport des céréales (sorgho,maïs) et/ou du pâturage naturel autre que la mangrove, contribue à une amélioration qualitative de cette ration de base. S'agissant d'une étude rétrospective, on n'a pas eu de précisions suffisantes sur le caractère saisonnier ou non de cette complémentation. On a donc considéré cette complémentation à l'échelle de l'année précédant l'interrogatoire des éleveurs (variable booléenne $\mathrm{O} / \mathrm{N}$ ). On peut alors considérer cette pratique comme hypothèse d'un facteur de "non-exposition" au risque de tomber "malade".

On peut ainsi définir ce facteur d'exposition par une variable synthétique appelée "ration" qui est égale à la somme de la présence de l'apport de céréale et/ou de pâturage naturel :

$$
\text { ration }=[(\mathrm{CR}=1)+(\mathrm{PT}=1)]
$$

tel que : $\mathrm{CR}=$ apport de céréales dans la ration, $\mathrm{PT}=$ pâturage naturel.

La variable ration a deux modalités : la première est l'absence totale de complémentation au fourrage de base (exposé au risque). La deuxième modalité est la présence d'au moins une des deux formes de complémentation décrites ci-dessus (non-exposé au risque).

Le croisement des deux variables synthétiques "malade" et "ration" donne un tableau de contingence 2 par 2 stratifié. Le nombre des strates est égal au nombre des classes d'âge des chamelons, soit dans le cas présent, deux classes : chamelons de moins de un an et chamelons de un à deux ans.

Pour garder l'homogénéité de l'échantillon, on a exclu de cette partie de l'analyse une exploitation qui ne distribue pas du tout de mangrove aux jeunes chamelons.

\section{RÉSULTATS DE L'ANALYSE}

\section{Description générale de l'élevage}

\section{Mode d'exploitation de la mangrove et performance zootechnique}

Dans l'échantillon, 61 p. 100 des éleveurs pratiquent un élevage mixte de camelins et petits ruminants. En moyenne, le troupeau camelin est constitué de dix à vingt-cinq individus. La mangrove peut être utilisée pour l'alimentation du dromadaire pendant toute l'année. Pour les autres espèces, c'est uniquement un fourrage de survie durant la sécheresse.

Les chamelons âgés de moins de un an pâturent avec leur mère dans la mangrove ou reçoivent des branches coupées sur place. Seules 30 p.100 des exploitations complémentent le fourrage de base (mangrove) avec des céréales et/ou par un pâturage sur parcours autre que la mangrove au moins une partie de l'année.

Lors du sevrage, les jeunes sont nourris avec des branches de mangrove dans les campements éloignés. Cette période est marquée par une augmentation du taux de mortalité chez les jeunes. Chez les femelles, la mise à la reproduction se fait à partir de l'âge de trois ans. La lactation dure généralement 360 jours. Soixante-dix pour cent des éleveurs estiment l'intervalle entre mises-bas à 18 mois environ. La réforme des femelles a lieu le plus souvent entre 14 et 20 ans.

\section{Problèmes sanitaires des chamelons}

On a répertorié quatre problèmes pathologiques majeurs chez les jeunes chamelons qui reçoivent de la mangrove : les maladies de peau (recensées dans 45 p.100 des élevages), les boiteries (dans 45 p.100 des campements également), les pertes d'appétit (27 p.100) et des paralysies des membres (20 p.100). En revanche, parmi les éleveurs qui ajoutent des céréales et/ou un temps de séjour sur pâturage naturel dans l'alimentation du troupeau, un sur sept seulement signale la présence de ces maladies (fig. 2). L'infestation par des ectoparasites (87 p.100 des cas), les piqûres de scorpion ainsi que les problèmes liés à la sous-alimentation sont, d'après les éleveurs, les causes premières de la mortalité des chamelons.

\section{Analyses multidimensionnelles}

On se limite à la prise en compte des trois premiers axes factoriels, nettement prépondérants. L'inertie expliquée par ces 3 premiers axes factoriels est respectivement de 46,4 p.100 pour le premier groupe de variables (utilisation de la mangrove), de 42,9 p.100 pour le deuxième 
nombre de campements

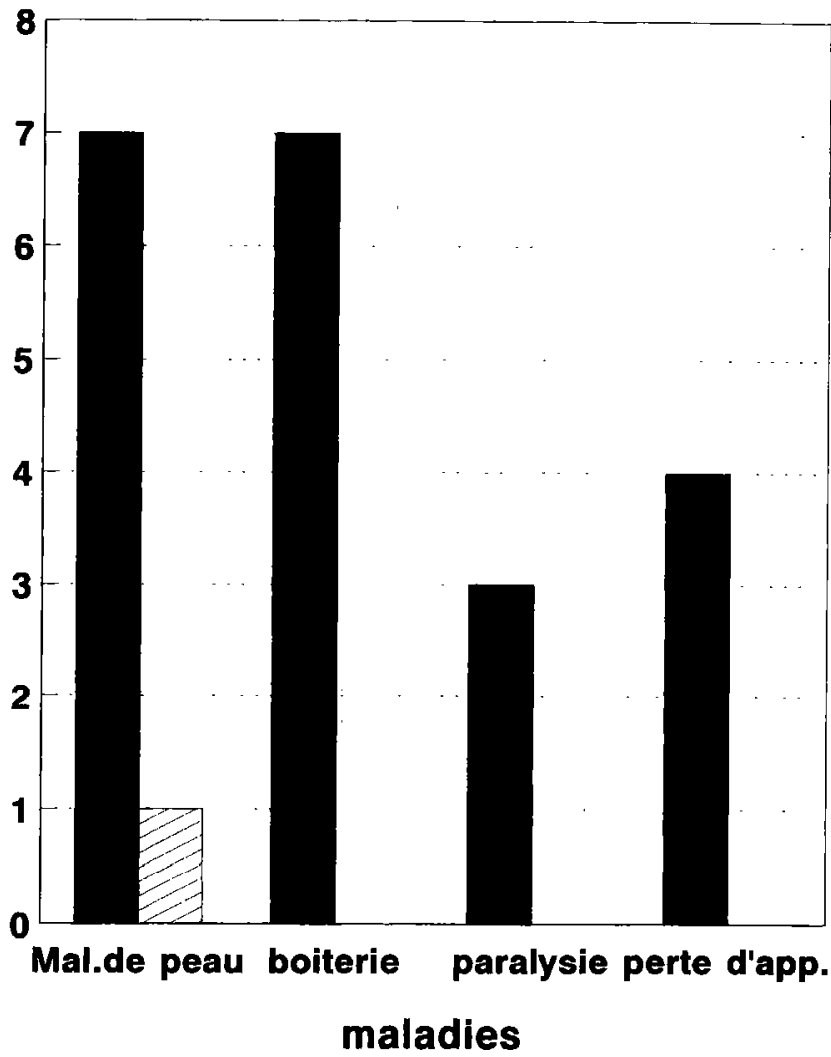

mangrove $(n=16) \quad$ mangrove+compl. $(n=7)$

Figure 2 : Occurrence des éleveurs ayant observé des pathologies chez le chamelon selon qu'ils distribuent ou non des compléments à la ration à base de mangrove.

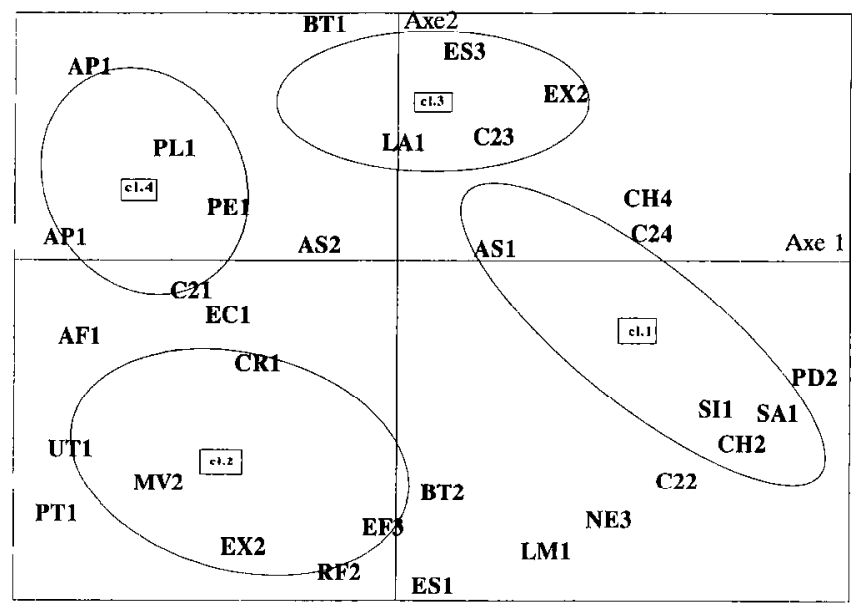

Figure 3 : Projection des variables les plus contributives au plan factorie $(1,2)$ et représentation graphique des quatre classes d'éleveurs retenues après classification des trois facteurs principaux. groupe (performances zootechniques et sanitaires) et de 37,5 p.100 pour l'analyse globale des deux groupes. La typologie est donc obtenue à partir de la classification de la combinaison des variables sur les trois premiers axes factoriels.

L'analyse permet d'isoler 4 classes (ou types) d'exploitations qui representent 65,8 p.100 de l'inertie totale (fig. $3)$. Ces 4 classes sont bien individualisées puisque la partition suivante n'apporte qu'un faible gain d'inertie $(7,8$ p.100).

La première classe est constituée de six éleveurs provenant des campements de Moulhoulé et Gahéré. Cette classe comprend l'éleveur qui ne distribue pas de mangrove aux jeunes chamelons. Les éleveurs font paître les chamelles dans la mangrove, les chamelons de moins de un an reçevant leur part dans les campements éloignés. Cette pratique d'élevage est associée à une mortalité précoce des chamelons due essentiellement à la sousalimentation. La mise à la reproduction des femelles se fait généralement avant trois ans.

La deuxième classe comprend trois éleveurs de Khor Anghar. Ce sont des éleveurs de grands troupeaux mixtes (29 dromadaires en moyenne). Toutes les espèces (camelin, bovin et petits ruminants) reçoivent de la mangrove comme fourrage de base complété de céréales et/ou d'un temps de parcours sur pâturage naturel autre que mangrove. Les problèmes liés aux "aphtes" sont déclarés comme causes majeures de mortalité chez les jeunes. La réforme des femelles adultes a lieu audelà de l'âge de vingt ans.

La troisième classe est formée de 9 éleveurs de 4 campements (Kibo, Gahéré, Khor Anghar et Lahassa). Cette classe est caractérisée par l'exploitation occasionnelle de la mangrove (pâturage de repli lors de sécheresse saisonnière). Les femelles allaitantes et les jeunes chamelons reçoivent des branches de mangrove coupées sur place. Les chamelons souffrent de boiterie.

La dernière classe est constituée de 5 éleveurs localisés dans trois campements : Lahassa, Gahéré et Khor Anghar. Pendant toute l'année, les dromadaires sont nourris exclusivement avec de la mangrove. Cette pratique est associée à la forte incidence des pathologies chez les jeunes (maladies de peau, perte d'appétit et paralysie des membres). En outre, la piqûre de scorpion est citée par l'éleveur comme cause principale de mortalité des jeunes.

\section{Estimation de l'association entre l'événement "malade" et l'exposition "mangrove"}

La force de l'association mesurée par l'odd-ratio standardisé est égale à 10,47. L'intervalle de confiance à $95 \mathrm{p}$. 100 est de $[1,49-119,24]$. Le risque relatif standardisé des maladies est égal à 4,28 . Les limites de confiance 
selon la méthode de GREENLAND-ROBINS sont $[1,16-$ $15,79]$. La valeur $\chi^{2}$ ajusté est très significative : $5,88(p=$ $0,01)$. La consommation de mangrove, en l'absence de toute complémentation constitue donc un facteur de risque des pathologies observées chez le chamelon.

\section{DISCUSSION}

Le faible nombre des variables décrivant les performances zootechniques, du fait de la présence de modalités rares, oblige à limiter la description plus détaillée du système d'élevage camelin. Le biais éventuel dans l'analyse est corrigé par l'annulation progressive des variables redondantes. Malgré la perte d'information, cela a permis de garder celles qui ont plus de signification dans l'interprétation générale.

Les maladies répertoriées dans la présente étude sont celles définies à partir des informations fournies par les éleveurs. II n'y a donc pas de diagnostic précis. On peut cependant suspecter que les "maladies de peau" englobent la gale et diverses dermatoses associées à la carence en zinc observée dans cette région (5). Les "boiteries" comprennent des troubles fonctionnels du pied, souvent marqués par un œdème des membres (2), dus à la sousalimentation. La notion de "manque d'appétit" est également associée à l'usage exclusif de la mangrove dont la teneur très riche en sel (5) déprime l'ingestibilité. Enfin les "paralysies" regroupent vraisemblablement des troubles locomoteurs profonds liés à des déficits alimentaires spécifiques (vitamines, minéraux), mais on n'en a pas de preuve formelle. Peut-on, par exemple, les imputer à une carence en cuivre induisant des formes d'ataxie néonatale chez les jeunes, troubles fréquemment observés sur les petits ruminants de la région $(4,5,7)$ ?

Dans une étude portant sur le statut nutritionnel du cheptel camelin de Djibouti, FAYE et MULATO (6) avaient déjà signalé un déficit énergétique et une carence en magnésium chez les dromadaires de la région d'Obock. Or, ces animaux sujets à divers troubles sanitaires, disposaient principalement de mangrove comme fourrage de base. Ici, on a pu remarquer que les éleveurs qui ne donnent pas de complément à la mangrove (céréales et/ou parcours sur pâturage naturel) se heurtent à de sérieux problèmes pathologiques chez les jeunes chamelons. Dans la plupart des cas, on y rencontre une fréquence élevée de troubles sanitaires.

La complémentation pratiquée à l'échelle du troupeau, soit par des céréales, soit par un pâturage sur des parcours naturels autre que la mangrove, est donc nécessaire pour éviter les troubles sanitaires observés. Le risque sanitaire chez les jeunes est en moyenne 4,28 fois plus élevé quand ils consomment exclusivement de la mangrove. Ces résultats confirment ceux observés en situa- tion expérimentale (8) : les troubles liés au déficit alimentaire associé à l'utilisation de la mangrove affectent essentiellement les jeunes animaux. En revanche, on n'observe pas nettement d'association entre les modes d'utilisation de la mangrove et les performances zootechniques relevées par les éleveurs. Le caractère rétrospectif de l'étude a, de fait, limité la fiabilité des réponses sur les critères globaux tels que l'intervalle entre mises-bas, à l'inverse des réponses concernant la santé qui relève d'événements plus facilement mémorisables. L'âge de la réforme, cependant, serait plus élevé dans le groupe assurant une complémentation au troupeau.

La variable (C21) qui indique la distribution des branches de mangrove dans les campements éloignés est fortement corrélée aux problèmes d'ectoparasitisme (EC1). L'accumulation des résidus de branches non consommés (seules les feuilles de mangrove sont ingérées par les animaux) près des enclos, constitue en effet un biotope favorable à la multiplication des tiques (2). L'abondance des tiques pourrait également expliquer la proximité des variables EC1 et PL1 (paralysie), des observations récentes ayant montré, chez le dromadaire, des cas de paralysie associés à une infestation massive par les tiques (16).

\section{CONCLUSION}

Une complémentation protéino-énergétique et minérale au fourrage de base est donc nécessaire pour assurer la survie et le développement des chamelons dans cette région. Une telle complémentation pourrait être assurée par l'apport de concentré en provenance du Service Aliment du Bétail (SAB) de Djibouti. Dans le cadre de notre enquête un seul éleveur pratiquait cette complémentation.

L'exploitation exclusive de la mangrove n'est pas du tout bénéfique pour le cheptel. D'une part, cette pratique favorise l'apparition de maladies chez les jeunes, d'autre part, elle peut complètement détruire cette formation végétale qui devient de plus en plus rare en République de Djibouti.

\section{REMERCIEMENTS}

Nous tenons à remercier le Ministère de la Coopération qui, à travers l'Unité de Coordination de l'Élevage Camelin (UCEC, responsable : Dr Gilles SAINT-MARTIN, CIRAD-EMVT) a permis la réalisation de cette étude dans le cadre du programme de recherche sur la pathologie nutritionnelle des camelidés à Djibouti, ainsi que le $\mathrm{Dr}$ KAMIL, chef du Service de l'Élevage de Djibouti qui nous a donné localement toutes les facilités nécessaires pour mettre en œuvre cette enquête. 


\section{B. Faye M. Ratovonanahary R. Cherrier}

\section{BIBLIOGRAPHIE}

1. AUDRU (J.), CÉSAR (J.), FORGIARINI (G.), LEBRUN (J.P.). La végétation et les potentialités pastorales de la République de Djibouti. Maisons-Alfort, IEMVT, 1987. 384 p.

2. CHERRIER (R.). Mise en place d'un essai d'alimentation chez le dromadaire à Obock. Mémoire de DESS de Productions animales en régions chaudes. Maisons-Alfort, CIRAD-IEMVT, 1990. 35 p.

3. ESCOFIER (B.), PAGES (J.). Mise en cuvre de l'analyse factorielle pour des tableaux numériques, qualitatifs ou mixtes. Rennes, IRISA, 1985. 56 p. (Publications Internes $n^{\circ} 263$ )

4. FAYE (B.), GRILLET (C.), TESSEMA (A.). Teneur en oligo-éléments dans les fourrages et le plasma des ruminants domestiques en Ethiopie. Revue Élev. Méd. vét. Pays trop.,1986, 39 (2) : 227-237.

5. FAYE (B.), KAMIL (M.), LABONNE (M.). Teneur en oligo-éléments dans les fourrages et le plasma des ruminants domestiques en République de Djibouti. Revue Élev. Méd. vét. Pays trop., 1990, 43 (3) : 365-373.

6. FAYE (B.), MULATO (C.). Facteurs de variation des paramètres protéoénergétiques, enzymatiques et minéraux dans le plasma chez le dromadaire de Djibouti. Revue Élev. Méd. vét. Pays trop., 1991, 44 (3) : 325-334.

7. FAYE (B.), GRILLET (C.), TESSEMA (A.), KAMIL (M.). Coppcr deficiency in ruminants in the Rift Valley of East Afrrica. Trop. Anim. Hlth Prod., 1991, 23:172-180.

8. FAYE (B.), SAINT-MARTIN (G.), CHERRIER (R.), RUFFA (A.). The influence of high dietary protein,energy and mineral intake on deficient young camel (Camelus Dromedarius). I. Changes in metabolic profiles and growth performance. Comp. Biochem. Physiol.,1992, 102A (2): 409-416.

FAYE (B.), RATOVONANAHARY (M.), CHERRIER (R.). Effect of a nutrition factor on the neonatal pathology. Results of a retrospective survey on the mangrove supply to dromedary calves in the Republic of Djibouti. Revue Élev. Méd. vét. Pays trop., 1993, 46 (3):471-478

The authors present a retrospective survey carried out in 23 camps of nomadic afars from Obock area (Djibouti) and concerning the traditional utilization of different types of forage from lagoon (mangrove) in relation with some of the diseases observed, particularly on calves. Four types of mangrove running are described: supply of mangrove twigs to calves less than one year old in camps and mangrove grazing for the adults; permanent feeding of mangrove associated with complementation; occasional supply of mangrove by cutting the twigs; permanent distribution of mangrove without complementation. The risk to observe diseases, namely loss of appetite, lameness, skin diseases and paralysis is $\mathbf{4 . 2 8}$ time higher when diet complementation is missing.

Key words : Dromedary - Camel calf - Feeding - Mangrove - Fodder Grazing - Supplementation - Pathological survey - Deficiency disease Skin disease - Lameness - Paralysis - Djibouti.
9. FAYE (B.). Mangrove, sécheresse et dromadaire. Science et changements planétaires. Sécheresse, 1993, 1 (4) : 47-55

10. FÉNELON (J.P.). Qu'est-ce que l'analyse des données? Paris, Lefonen Éd, $1981.311 p$

11. GODET (J.). Notes sur l'élevage camelin en République de Djibouti. Djibouti, ISERST, 1985. P. 32-39.

12. JENICEK (M.), CLEROUX (R.). Épidémiologie, Principes, Techniques, Applications. Ste Hyacinthe (Québec), EDISEM Ed., 1987. P. 143203.

13. LEBEAUX (M.O.). Manuel de réference ADDAD (Association pour la Diffusion et le Développement de l'Analyse de Données). Paris, ADDAD. 1985.

14. METHA (C.), PATEL (N.), GRAY (R.). A method for the estimation of the tree confident interval of odd-ratio. J. Am. statist. Ass. 1985, $\mathbf{7 8}$ 969-973.

15. MULATO (C.). Profil métabolique et statut nutritionnel camelins dans la République de Djibouti. Mémoire de DESS de Productions animales en régions chaudes. Maisons-Alfort, CIRAD-IEMVT, 1989. $141 \mathrm{p}$

16. MUSA (M.T), OSMAN (O.M.). An outbreak of suspected tick paralysis in one-humped camels (Camelus dromedarius) in the Sudan. Revue Élev. Méd. vét. Pays trop., 1990, 43 (4) : 505-510

17. ROTHMAN (K.J.), BOICE (J.). Epidemiologic analysis with a programmable calculator. Washington, US. Dept. Health Educ., Publ. Health Service, NIH Publ., 1979. 1649 p.

18. THRUSFIELD (M.). Veterinary Epidemiology. Kent, England, Publ. Butterworth \& Co., 1986. 280 p.

FAYE (B.), RATOVONANAHARY (M.), CHERRIER (R.). Efecto de un factor alimenticio sobre la patología neonatal : Resultados de una encuesta retrospectiva sobre la distribución de mangle a camellejos de la República de Djibuti. Revue Élev. Méd. vét. Pays trop., 1993, 46 (3) : $471-478$

Se llevó a cabo una encuesta retrospectiva en 23 campamentos nómadas de la región de Obock (Djibuti), con el fin de estudiar el uso tradicional de la vegetación lagunar (mangle) y su relación con las patologías observadas, principalmente en camellos jóvenes. Se pueden describir cuatro tipos de explotación del manglar : distribución de ramas de mangle a los camellejos menores de un año y pastoreo de los adultos en el manglar ; uso permanente del manglar, con complementación ; explotación ocasional mediante podas ; uso permanente sin complementación. En los casos de raciones a base de manglar, sin complementación, se observó un riesgo 4,28 veces más elevado de problemas de salud (pérdida de apetito, renqueras, parálisis, enfermedades de la piel).

Palabras calves : Dromedario - Camellejo - Alimentación - Mangle Forrajc - Pastoreo - Complementación - Encuesta patológica - Carencia Enfermedad de la piel - Cojero - Parálisis - Djibuti. 